\title{
High fidelity modeling of SRP and its effect on the relative motion of Starshade and WFIRST
}

\author{
Ariadna Farres * $\quad$ Cassandra Webster ${ }^{\dagger}$ \\ Dave Folta ${ }^{\ddagger}$ NASA Goddard Space Flight Center, Greenbelt, Maryland, 20771, United States
}

\begin{abstract}
In this paper we perform a detailed analysis of how Solar Radiation Pressure (SRP) affects the relative motion of two spacecrafts, the Wide-Field Infrared Survey Telescope (WFIRST) and Starshade, orbiting in the vicinity of the Sun-Earth L2. While WFIRST orbits about its own Libration Point Orbit (LPO), Starshade will fly a specific trajectory to align with WFIRST and observe a Design Reference Mission of pre-determined target stars. In this analysis, we focus on the transfer orbit for Starshade from one observation to the other. We will describe how SRP affects the dynamics of the Starshade relative to WFIRST and how relevant this effect is in order to get an accurate estimate of the total $\Delta v$.
\end{abstract}

\section{Introduction}

The Wide-Field Infrared Survey Telescope (WFIRST) is a NASA observatory designed to answer questions about dark energy and astrophysics, ${ }^{1}$ that is planning to fly in a Libration Point orbit (LPO) around the Sun-Earth $L_{2}$ (SEL2). WFIRST will use a 2.4 meter mirror (the same size as the Hubble Space Telescope's) along with a Wide-Field Instrument (WFI) and a Coronagraph Instrument (CGI) to achieve its mission objectives. While the primary objective of the Coronagraph Instrument is to search for exoplanets, the use of an external occulter such as Starshade ${ }^{2}$ would make the detection of Earth-sized planets in habitable zones of nearby stars possible.

WFIRST will answer questions about dark energy, exoplanets, and infrared astrophysics, and is planned to launch in 2026. The WFI will have a field of view 100 times greater than Hubble's infrared instrument, which will allow WFIRST to capture more area of the sky in less time. With its large field of view, the WFI will be able to measure light from a billion galaxies over the WFIRST mission lifetime, and it will perform a survey of the inner Milky Way using microlensing to find thousands exoplanets. The CGI will be used to perform high contrast imaging and spectroscopy of closer exoplanets. The CGI will use internal occulting through different mirrors, lenses, and masks to filter starlight and image gas-giant planets and possibly super-Earths. The ability to directly image another Earth-like planet, however, will not be done by the CGI alone since it does not have the contrasting power. However, the CGI combined with an external Starshade, is believed to provide the high contrast required to image an Earth-2.0 decades ahead of time. Starshade is a 34 meter flower shaped occulter with razor-sharp petals that would be designed to redirect diffraction from a star's light (which would produce an undesirable glare) and create a shadow for WFIRST to enter for an exoplanet observation using its CGI. Many researchers believe that Starshade along with the CGI on WFIRST would allow for a direct image of an Earth 2.0. Starshade would be launched several years after WFIRST, and would trail WFIRST in its LPO at SEL2. After some time, Starshade would perform a maneuver to place itself in a different LPO that is $37,000 \mathrm{~km}$ or more away from WFIRST. At this point, Starshade can begin slewing directly between WFIRST and a target star to attempt exoplanet observation. Starshade would attempt to achieve all targeted observations over 2 years. Figure 1 shows the telescope/Starshade observation concept.

Given that the SEL2 environment is very unstable, WFIRST will need to perform routine Station keeping maneuvers to maintain its nominal mission orbit. WFIRST will utilize a Quasi-Halo orbit to navigate the

*Visiting Researcher, Goddard Planetary Heliophysics Institute, University of Maryland Baltimore County, Baltimore, MD

$\dagger^{\dagger}$ FIRST Flight Dynamics Lead, NASA Goddard Space Flight Center, Greenbelt, MD

${ }^{\ddagger}$ Senior Fellow Chair, NASA Goddard Space Flight Center, Greenbelt, MD 


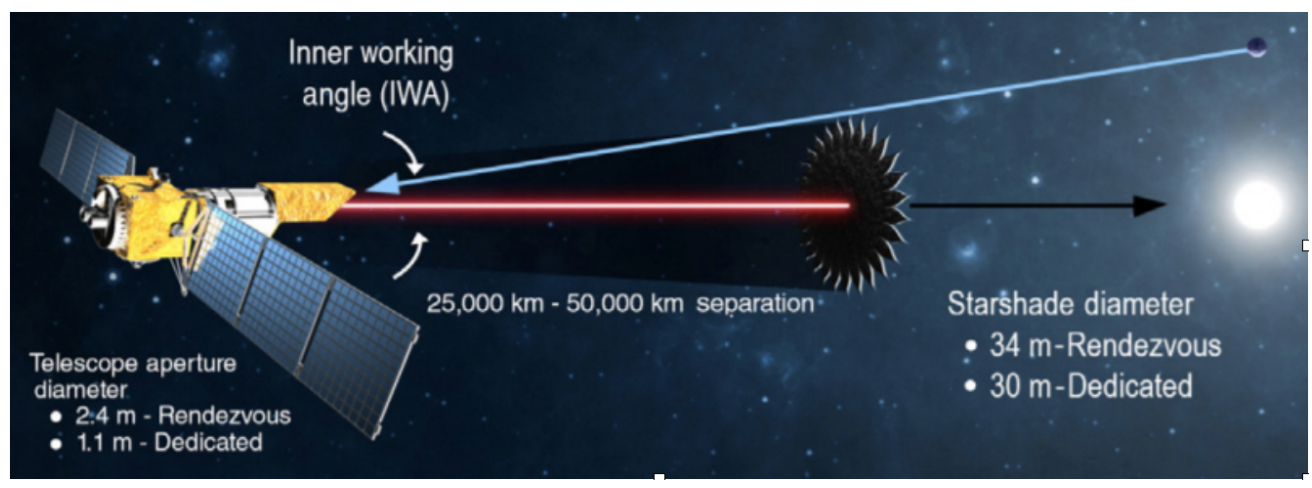

Figure 1. Representation of the Telescope/Starshade Concept

SEL2 environment, while Starshade will need to fight against the natural dynamics and the effects of Solar Radiation Pressure (SRP) in order to achieve the desired exoplanet observations. ${ }^{4}$ While WFIRST will be in its mission LPO, Starshade will fly a specific trajectory to align with WFIRST and observe a Design Reference Mission (DRM $)^{3}$ of pre-determined target stars. A recent study was undertaken at NASA's Goddard Space Flight Center (GSFC) to determine the compatibility of flying Starshade with WFIRST. This preliminary study investigated targeting methods to estimate the $\Delta V$ budget to transfer Starshade from one observation alignment to the next, while following a desired observation sequence. ${ }^{9}$

The challenges of formation transfers at SEL2 include achieving the observation's location with respect to WFIRST for each target star, while also managing the unstable SEL2 environment. Starshade will essentially operate like a solar sail and will undergo large perturbations due to SRP which will affect Starshade's overall $\Delta V$ budget for operations. Orbit maintenance maneuvers will be required in order to keep both WFIRST and Starshade in the SEL2 environment. Additionally, WFIRST will need to perform routine Momentum Unloads (MUs) to unload stored momentum in the reaction wheels.

In this paper, we analyze the effects of SRP on both WFIRST and Starshade by considering different SRP models. We want to understand how SRP affects the dynamics of the system for both spacecraft and what implications this has on their relative motion. We will mainly focus on transfer orbits for Starshade from one observation to another. Given a specific DRM with a specific set of stars to observe, Starshade must change its relative position with respect to WFIRST with a specific time of flight to observe a different star. The time of flight will also be specified in the DRM. The preliminary analysis ${ }^{9}$ showed the relevance of the dynamics on the final $\Delta V$ budget. There we saw that by changing the starting date of the DRM by one month, the $\Delta V$ required to transfer to a specific observations changed drastically. The investigations in this previous model considered the classical cannonball model with the same SRP coefficient for both spacecraft. This has encouraged us to continue studying this problem including a higher fidelity model for the SRP. By modeling a high fidelity SRP for both WFIRST and Starshade, we can provide insight into how to plan the observations for Starshade minimizing the total $\Delta V$ for each observation and thereby maximizing Starshade's mission lifetime.

This paper is organized as follows: in section II we describe the equations of motion and the different SRP models that we have used. In section III we describe how SRP affects the dynamics around a Halo orbit. There we will introduce the Floquet model reference frame that will help us describe the motion around LPOs. In section IV we present WFIRST's nominal dynamics and make some comments on the Station Keeping strategy that will be used. Finally in section V we analyze the effects of SRP on the formation and transfer trajectories of Starshade around WFIRST between observations. We will finish with some conclusions and possible extensions for this study.

\section{Dynamical Model}

To have a general idea on the complexity of the problem and perform a preliminary analysis on the relevance of SRP in the formation flying of WFIRST and Starshade, we use the Sun-Earth Restricted Three Body Problem (RTBP) as a model. The RTBP has proven to be a good model for preliminary mission analysis at LPO's as it includes the most relevant effects in the Sun-Earth $L_{1}$ (SEL1) and SEL2 environments. In 
Fig. 2 we have a schematic representation on the different forces that are included in this model.

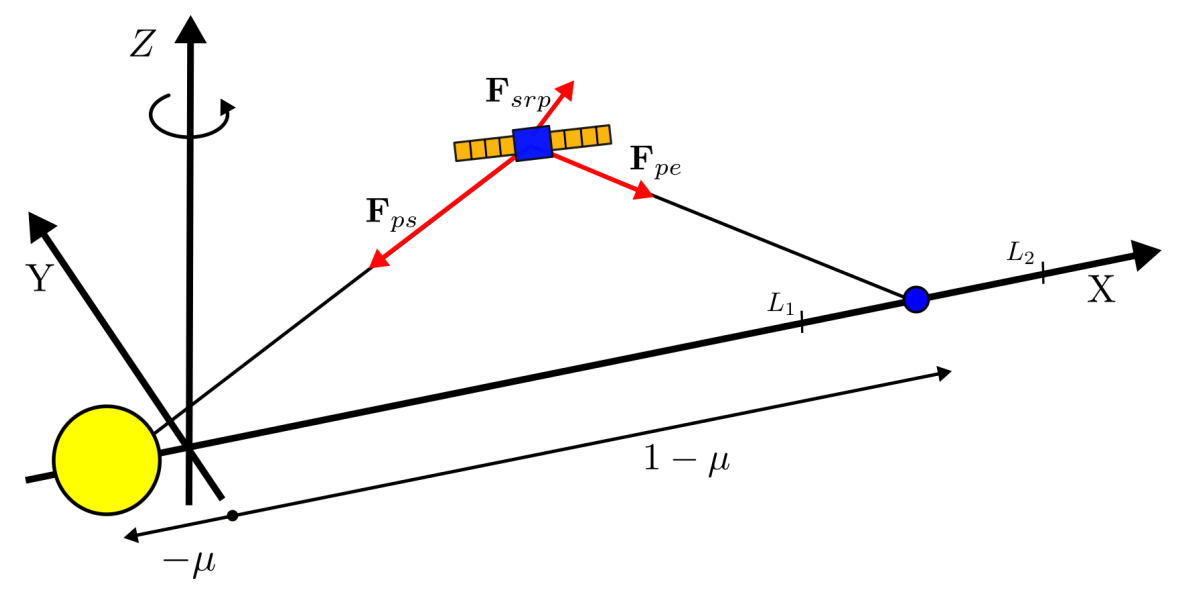

Figure 2. Schematic representation for the RTBP and the different forces that are included in our equations of motion.

\section{A. Restricted Three Body Problem}

We recall that the RTBP considers that both, Earth and Sun, are point masses which orbit around their common center of mass in a circular way due to their mutual gravitational attraction. The spacecraft, on the other hand, is a mass-less particle that is affected by the gravitational attraction of the two primaries but does not affect their motion. The units of mass, distance and time have been normalized such that the total mass of the system is 1, the Earth-Sun distance is 1 and the period of one Earth-Sun revolution in $2 \pi$. With these normalized units the universal gravitation constant $G=1$ and Earth's mass is $\mu=3.040423402 \times 10^{-6}$ (i.e. $(1-\mu)$ is the Sun's mass). Moreover, a rotating reference frame is considered, where the origin is at the Earth-Sun center of mass and the two primaries are fixed on the $\mathrm{x}$-axis (with the positive side pointing towards the Earth); the z-axis is perpendicular to the ecliptic plane and the $\mathrm{y}$-axis completes an orthogonal positive oriented reference frame. With these assumptions and including the SRP acceleration, the equations of motion are:

$$
\ddot{X}-2 \dot{Y}=\frac{\partial \Omega}{\partial X}+a_{x}, \quad \ddot{Y}+2 \dot{X}=\frac{\partial \Omega}{\partial Y}+a_{y}, \quad \ddot{Z}=\frac{\partial \Omega}{\partial Z}+a_{z},
$$

where $\Omega(X, Y, Z)=\frac{1}{2}\left(X^{2}+Y^{2}\right)+\frac{(1-\mu)}{r_{p s}}+\frac{\mu}{r_{p e}}, \mathbf{a}_{s r p}=\left(a_{x}, a_{y}, a_{z}\right)$ represents the acceleration due to SRP, and $r_{p s}=\sqrt{(X+\mu)^{2}+Y^{2}+Z^{2}}, r_{p e}=\sqrt{(X+\mu-1)^{2}+Y^{2}+Z^{2}}$ are the Sun-satellite and Earth-satellite distances respectively.

\section{B. Solar Radiation Pressure}

Solar Radiation Pressure is the acceleration due to the exchange in momenta between the photons emitted by the Sun and the satellite's surface. The incident light will be both absorbed and reflected by the surface of the satellite. The rate of absorption $\left(\rho_{a}\right)$ and reflection $\left(\rho_{s}, \rho_{d}\right)$ will depend on the properties of the surface material. Hence, the total acceleration due to SRP will vary depending on the shape of the satellite, the material's reflectivity properties of the different components, and its relative orientation with respect to the Sun-satellite line.

It is true that this extra acceleration is very small compared to the gravitational attraction of the two primaries and other perturbations in space. However, as we will see, it plays an important role in the dynamics at LPO's and its effect must be take into account. There are several models, depending on the level of fidelity required. In this section we briefly describe each of them.

\section{Cannonball model}

The cannonball model is the simplest and most common approach used to model the SRP acceleration, where we approximate the shape of the satellite by a sphere. ${ }^{10}$ In this case the SRP acceleration, $\mathbf{a}_{s r p}$, is 
always in the satellite-Sun direction $\mathbf{r}_{s}$, and can be expressed as:

$$
\mathbf{a}_{\text {srp }}=-\frac{P_{\text {srp }} C_{r} A_{\text {sat }}}{m_{\text {sat }}} \mathbf{r}_{\mathbf{s}},
$$

where $C_{r}=2\left(1-\rho_{s}\right)$ is the reflectivity coefficient, $P_{\text {srp }}=P_{0}\left(R_{0} / R_{\text {sun }}\right)^{2}\left(\right.$ where $P_{0}=4.57 \times 10^{-6} \mathrm{~N}$ is the $\mathrm{SRP}$ at $R_{0}=1 \mathrm{AU}$, and $R_{\text {sun }}$ is the distance to the Sun), $A_{\text {sat }} / m_{\text {sat }}$ is the satellite's area-to-mass ratio, and $\mathbf{r}_{s}$ is the normalized Sun-satellite direction (i.e. in the RTBP reference frame $\left.\mathbf{r}_{s}=(X-\mu, Y, Z) / r_{p s}\right)$.

Notice that $P_{s r p}$ depends on the inverse of the Sun-satellite distance in the same way as the Sun's gravitational attraction. Hence in the RTBP context it is common to define the parameter $q_{\text {srp }}$, the ratio between the SRP acceleration and the Sun's gravity. And Eq. 2 can be rewritten as:

$$
\mathbf{a}_{s r p}=-q_{s r p} C_{r} \frac{(1-\mu) \mathbf{r}_{s}}{r_{p s}^{2}}
$$

where $q_{\text {srp }}=\left(P_{0} R_{0}^{2} / G m_{\text {sun }}\right)\left(A_{\text {sat }} / m_{\text {sat }}\right)=7.7065 \times 10^{-4}\left(A_{\text {sat }} / m_{\text {sat }}\right)$ when $A_{\text {sat }}$ is given in $\mathrm{m}^{2}$ and $m_{\text {sat }}$ is given in $\mathrm{kg}$.

With this we can merge the SRP acceleration with the Solar gravitational attraction in Eq.1 and rewrite $\Omega(X, Y, Z)$ as $\tilde{\Omega}(X, Y, Z)=\frac{1}{2}\left(X^{2}+Y^{2}\right)+\frac{\left(1-q_{s r p} C_{r}\right)(1-\mu)}{r_{p s}}+\frac{\mu}{r_{p e}}$.

\section{N-plate model}

The N-plate model approximates the shape of the satellite by a collection of flat plates, each of them having different reflectivity properties representing the different sides of the satellite. In this approach the magnitude of the SRP acceleration will vary depending on the satellite's orientation with respect to the Sun.

If we consider a flat surface, the total force due to SRP is the sum of the force produced produced by: the photons that are absorbed $\left(\mathbf{F}_{\mathbf{a}}=P_{s r p} A\left\langle\mathbf{n}, \mathbf{r}_{\mathbf{s}}\right\rangle \mathbf{r}_{\mathbf{s}}\right)$ and the photons that are reflected, which experience both: specular reflection $\left(\mathbf{F}_{s}=2 P_{s r p} A\left\langle\mathbf{n}, \mathbf{r}_{\mathbf{s}}\right\rangle^{2} \mathbf{n}\right)$ and diffusive reflection $\left(\mathbf{F}_{\mathbf{d}}=P_{s r p} A\left\langle\mathbf{n}, \mathbf{r}_{\mathbf{s}}\right\rangle\left(\mathbf{r}_{\mathbf{s}}+\frac{2}{3} \mathbf{n}\right)\right)$.

We define the coefficients $\rho_{a}, \rho_{s}, \rho_{d}$ as the rates of absorption, specular and diffusion, which depend on the properties of the surface material, and satisfy $\rho_{a}+\rho_{s}+\rho_{d}=1$ (i.e. $\rho_{a}=1-\rho_{s}-\rho_{d}$ used to reduce the number of coefficients). Hence, for a flat plate the total SRP acceleration is given by:

$$
\mathbf{a}_{s r p}=-\frac{P_{s r p} A}{m_{s a t}}\left\langle\mathbf{n}, \mathbf{r}_{\mathbf{s}}\right\rangle\left[\left(1-\rho_{s}\right) \mathbf{r}_{\mathbf{s}}+2\left(\rho_{s}\left\langle\mathbf{n}, \mathbf{r}_{\mathbf{s}}\right\rangle+\frac{\rho_{d}}{3}\right) \mathbf{n}\right],
$$

where $\mathbf{r}_{s}$ is the normalized satellite-Sun direction and $\mathbf{n}$ is the normal direction to the flat surface pointing away from the Sun. Again we can rewrite $P_{s r p}\left(A / m_{s a t}\right)$ as $q_{s r p}(1-\mu) /\left(r_{p} s^{2}\right)$.

If we define our satellite as a collection of $N$ plates, where each plate has its own area $A_{k}$, reflectivity properties $\rho_{s}^{k}, \rho_{d}^{k}$ and a relative orientation with respect to the Sun $\mathbf{n}_{k}$, the total SRP acceleration is given by adding $N$ copies Eq. 4 one for each plates. Further details on its implementation can be found in Ref. 11.

\section{High-Fidelity approach}

The main drawback of the $N$-plate model is that it does not take into account possible auto occultation between the different plates when the satellite's attitude varies along its orbit. In order to take into account the auto occultation between the different satellite components Ziebart ${ }^{7,8}$ proposed to use ray-tracing techniques to determine which parts of the satellite are illuminated for certain orientations with respect to $\mathbf{r}_{\mathbf{s}}$. In order to have an accurate approximation for the SRP we need a good CAD model and a large number of points on what they call pixel-array (used during the ray-tracing computations). Unfortunately this method is very expensive in terms of computational time and it is not advisable to compute this simultaneously during an orbit simulation. In order to optimize its performance one must know the attitude profile in advance and compute the SRP acceleration for each one on the profile, or approximate its value from a set of intermediate attitudes.

For further details on the implementation of these method check the Thesis by Ziebart ${ }^{5}$ and Rivers. ${ }^{6}$ Moreover, in Farres ${ }^{11}$ you can find a comparison between these three different SRP models. 


\section{Coupled WFIRST and Starshade dynamics}

As we have already mentioned, in this paper we focus on the formation flying of WFIRST and Starshade, which are required to remain close to each other while orbiting around a Halo or Quasi-Halo orbit at SEL2. During the Coronagraph observations Starshade must remain 37,000 km from WFIRST in a specific geometric configuration pointing towards the target star.

From a computational point of view, we can think of this problem as two coupled RTBP, one for each satellite, where Starshade decides its position relative to WFIRST who will perform station keeping maneuvers to remain close to a nominal Halo orbit. We note that the area-to-mass ratio and the reflectivity properties of both satellites are very different, so SRP will affect each of them in a different way. From a dynamical point of view we also have two coupled RTBP with different SRP coefficients.

In this paper we consider a cannonball model for the SRP effect on WFIRST and both the cannonball and the N-plate models for Starshade. By doing this, we want to see the impact that changes on the orientation of Starshade with respect to the Sun-line have on the total $\Delta v$ cost. WFIRST has an estimated area-to-mass ratio of $0.00675 \mathrm{~m}^{2} / \mathrm{kg}$ while Starshade's estimated area-to-mass ratio is $0.5543 \mathrm{~m}^{2} / \mathrm{kg}$. Hence the SRP coefficients $\left(q_{\text {srp }}\right)$ are $1.0 \times 10^{-5}$ and $8.5 \times 10^{-4}$ for WFIRST and Starshade respectively.

\section{Impact of Solar Radiation Pressure on the RTBP dynamics}

It is well known ${ }^{12,13}$ that when we include the SRP effect on the RTBP, the five equilibrium points of the system are displaced towards the Sun. The same happens with the different families of periodic and quasi-periodic orbits of the system. For small $q_{s r p}$ values the qualitative behavior of the system is the same.

Table 1 shows the position of the SEL2 point for different $q_{s r p}$ values. It can be seen that there is a difference of more than $13,000 \mathrm{~km}$ between the location of the displaced SEL2 for Starshade $\left(q_{s r p}=8.5 \times 10^{-4}\right)$ and WFIRST $\left(q_{s r p}=1.0 \times 10^{-5}\right)$.

Table 1. Location of the displaced $L_{2}$ point when including the effect of SRP. Notice that $q_{s r p}=1.0 \mathrm{e}-05$ is close to WFIRST's SRP and $q_{s r p}=8.5 \mathrm{e}^{-4}$ is close to Starshade's SRP value.

\begin{tabular}{|c|c|c|}
\hline$q_{s r p}$ & $L_{2}(\mathrm{AU})$ & $L_{2}(\mathrm{~km})$ \\
\hline \hline 0.0 & $(1.010075200020 \mathrm{e}+00,0.00 \mathrm{e}+00,0.00 \mathrm{e}+00)$ & $(151105099.16996,0.0,0.0)$ \\
$5.0 \mathrm{e}-06$ & $(1.010074648234 \mathrm{e}+00,0.00 \mathrm{e}+00,0.00 \mathrm{e}+00)$ & $(151105016.62386,0.0,0.0)$ \\
$1.0 \mathrm{e}-05$ & $(1.010074096507 \mathrm{e}+00,0.00 \mathrm{e}+00,0.00 \mathrm{e}+00)$ & $(151104934.08668,0.0,0.0)$ \\
$5.0 \mathrm{e}-05$ & $(1.010069684836 \mathrm{e}+00,0.00 \mathrm{e}+00,0.00 \mathrm{e}+00)$ & $(151104274.11009,0.0,0.0)$ \\
$1.0 \mathrm{e}-04$ & $(1.010064175608 \mathrm{e}+00,0.00 \mathrm{e}+00,0.00 \mathrm{e}+00)$ & $(151103449.94131,0.0,0.0)$ \\
$5.0 \mathrm{e}-04$ & $(1.010020315436 \mathrm{e}+00,0.00 \mathrm{e}+00,0.00 \mathrm{e}+00)$ & $(151096888.55297,0.0,0.0)$ \\
$8.5 \mathrm{e}-04$ & $(1.009982247617 \mathrm{e}+00,0.00 \mathrm{e}+00,0.00 \mathrm{e}+00)$ & $(151091193.68830,0.0,0.0)$ \\
\hline
\end{tabular}

We have computed, for different $q_{s r p}$ values, the families of displaced Halo orbits. On the left-hand side of Figure 3 we have the intersection of these orbits with the Poincaré section $\{Y=0, \dot{Y}>0\}$. We note that each point on the plot corresponds to one periodic orbit. In this plot we can appreciate how the periodic orbits move towards the Sun as $q_{s r p}$ increases. Notice that between the Halo orbits for WFIRST $\left(q_{s r p}=1.0 \times 10^{-5}\right)$ and Starshade $\left(q_{s r p}=8.5 \times 10^{-4}\right)$ there is at least 7,000 km of separation. On the right-hand side of Figure 3 we have the relation between the $Z$ amplitude of these orbits on the Poincaré section and their orbital period. Here we can see that between the SRP values for WFIRST and Starshade the difference in the orbital period of the Halo orbits is around 2 days.

This means that the natural dynamics for each probe in the SEL2 environment will different as each one of them will be governed by the linear and non-linear dynamics of different periodic orbits, that are displaced from each other and have different orbital periods. By imposing the requirement that Starshade and WFIRST maintain a certain relative distance, we are forcing Starshade to follow an unnatural path which can increases the $\Delta V$ to do operations. 

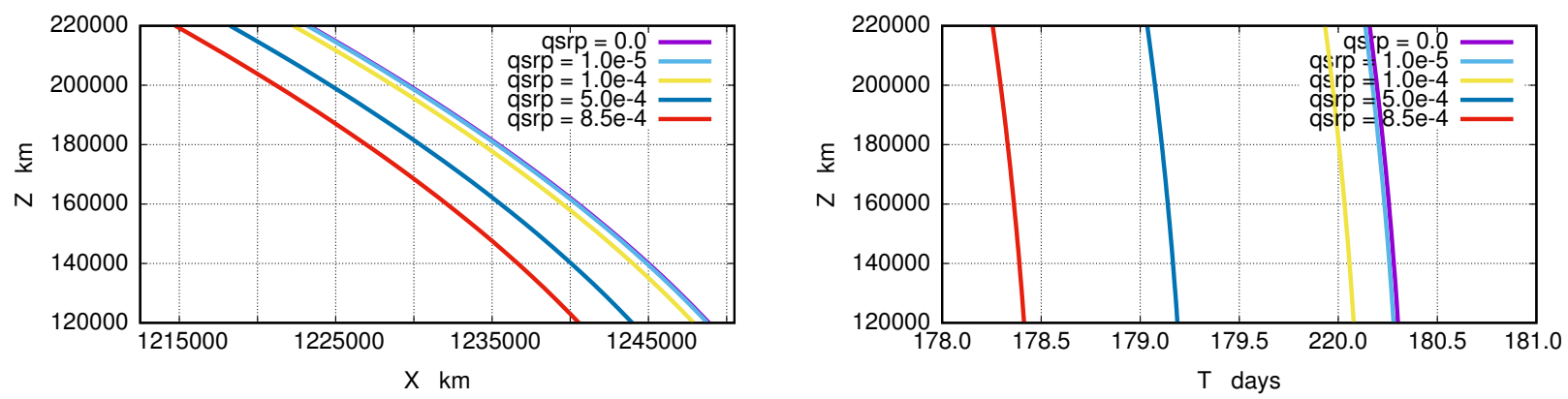

Figure 3. For different $q_{s r p}$ values, family of Halo orbits where $Z_{\max } \in[120000,220000] \mathbf{k m}$. Left: $Z$ vs $X$ on the $Y=0$ Poincaré section, Right: $Z$ vs $T$ the orbits period.

\section{A. Linear dynamics around Halo orbits}

The local behavior around a periodic orbit is given by the first order variational equations. To fix notation, let $\phi$ be the flow associated to the equations of motion. Where, $\phi_{\tau}\left(x_{0}\right)$ is the image of the point $x_{0} \in \mathbb{R}^{6}$ at time $t=\tau$, and $A(\tau)=D \phi_{\tau}\left(x_{0}\right)$ is the first order variational of $\phi_{\tau}\left(x_{0}\right)$ with respect to the initial condition, $x_{0}$. Hence, $\phi_{\tau}\left(y_{0}\right)+A(\tau) \cdot h$, the linear approximation of $\phi_{\tau}(y 0+h)$, gives a good approximation provided $h \in \mathbb{R}^{6}$ is small.

The linear dynamics around a periodic orbit, can be described through the study of the eigenvalues and eigenvectors of the state transition matrix $A(T)$, where $T$ is the period of the orbit. One can check that for all the Halo orbits considered here the eigenvalues $\left(\lambda_{1, \ldots, 6}\right)$ of $A(T)$ always satisfy: $\lambda_{1}>1, \lambda_{2}<1, \lambda_{3}=\bar{\lambda}_{4}$ and have modulus one, and $\lambda_{5}=\lambda_{6}=1$. These three pairs of eigenvalues and their associated eigenvectors have the following geometrical meaning: ${ }^{14}$

- The first pair $\left(\lambda_{1}, \lambda_{2}\right)$, verify $\lambda_{1} \cdot \lambda_{2}=1$, and are related to the hyperbolic character of the orbit. The value $\lambda_{1}$ is the largest in absolute value, and is related to the unstable eigenvalue $e_{1}(0)$, the most expanding direction. After one period, an distance $d_{0}$ to the nominal orbit in the direction $e_{1}(0)$ is amplified by a factor of $\lambda_{1}$. Using $D \phi_{\tau}$ we can get the image of this vector under the variational flow: $e_{1}(\tau)=D \phi_{\tau} e_{1}(0)$, which together with the vector tangent to the orbit span a plane tangent to the local unstable manifold. In the same way $\lambda_{2}$ and its related eigenvector $e_{2}(0)$ are related to the stable manifold and $e_{2}(\tau)=D \phi_{\tau} e_{2}(0)$. The state transition matrix restricted to the plane generated by $e_{1}(0), e_{2}(0)$ is:

$$
\left(\begin{array}{rr}
\lambda_{1} & 0 \\
0 & \lambda_{2}
\end{array}\right)
$$

- The second pair $\left(\lambda_{3}, \lambda_{4}\right)$ are complex conjugate eigenvalues of modulus 1 . The monodromy matrix restricted to the plane spanned by $e_{3}(0)$ and $e_{4}(0)$ (the real and imaginary parts of the eigenvectors associated to $\left.\lambda_{3}, \lambda_{4}\right)$ is a rotation of angle $\Gamma=\arctan \left(\frac{\operatorname{Im}\left(\lambda_{3}\right)}{\operatorname{Re}\left(\lambda_{3}\right)}\right)$ along the orbit. The state transition matrix restricted to the plane generated by $e_{3}(0), e_{4}(0)$ is of the form

$$
\left(\begin{array}{rr}
\cos \Gamma & -\sin \Gamma \\
\sin \Gamma & \cos \Gamma
\end{array}\right) .
$$

- The third couple $\left(\lambda_{5}, \lambda_{6}\right)=(1,1)$, is associated to the neutral directions. $A(T)$ only has one eigenvector with eigenvalue equal to 1 , and this vector is the tangent vector to the orbit that we call $e_{5}(0)$. The other eigenvalue is associated to variations of the period, or any other variable that parameterize the family of periodic orbits. The related eigenvector is taken orthogonal to $e_{5}(0)$ in the $2 \mathrm{D}$ space associated to the eigenvalue 1 , and gives the tangent direction to the family of Halo orbits. The monodromy matrix restricted to this plane has the form

$$
\left(\begin{array}{ll}
1 & \varepsilon \\
0 & 1
\end{array}\right) .
$$

The fact that $\varepsilon$ is not zero is due to the variation of the orbits period as we move along the family. 
The functions $e_{i}(\tau)=D \phi_{\tau} \cdot e_{i}(0), i=1, \ldots, 6$, gives an idea of the variation of the phase space properties in a small neighborhood of the periodic orbit. It is convenient to use the Floquet modes $\bar{e}_{i}(\tau), i=1,6$, six $T$-periodic functions that can easily be recovered by $e_{i}(\tau)$, to define a reference system around a Halo orbit. One of the advantages of the Floquet modes is that they are periodic and can be spanned as a Fourier series and easily stored by their Fourier coefficients. For further details on the computation and use of the Floquet modes see Ref. 14.

Using the Floquet mode reference frame, the dynamics around a Halo orbit is simple: on the planes generated by $\bar{e}_{1}(\tau), \bar{e}_{2}(\tau)$ the trajectory will escape with an exponential rate along the unstable direction $\left(\bar{e}_{1}(\tau)\right)$; on the planes generated by $\bar{e}_{3}(\tau), \bar{e}_{4}(\tau)$ the dynamics consists of a rotation around the periodic orbit; and on the planes generated by $\bar{e}_{5}(\tau), \bar{e}_{6}(\tau)$ the dynamics is neutral. In Figure 4 we have a schematic representation of the linear dynamics around the orbit, where the fixed point corresponds to the periodic orbit.
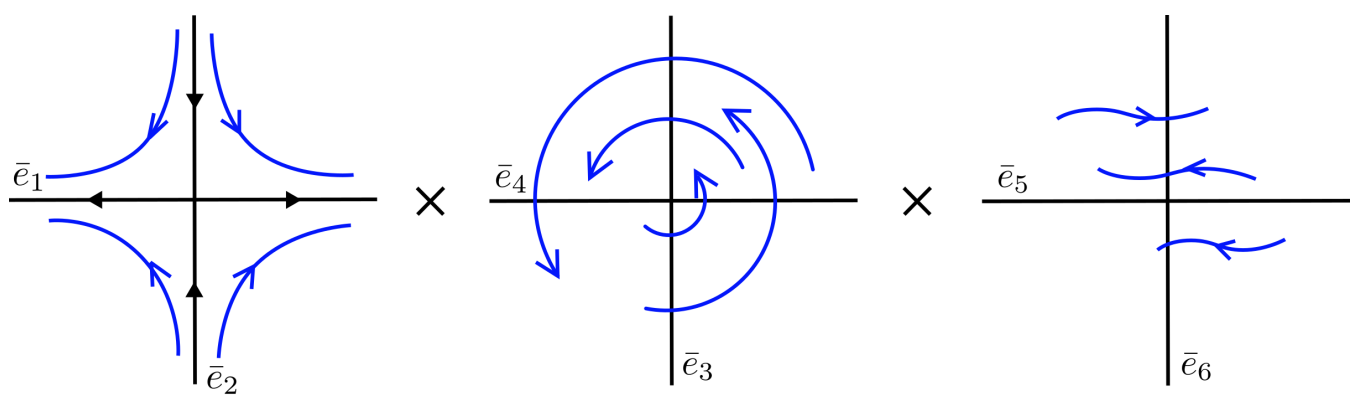

Figure 4. Schematic representation of the linear dynamics around a Halo orbit using the Floquet modes $\bar{e}_{i}(\tau)$.

This reference frame not only allows us to have a good description of the motion on the satellite close to a Halo orbit, but as we will see in Section IV it can also be used to derive station keeping maneuvers.

\section{B. Displaced Halo orbit in the Floquet mode reference frame}

As we have mentioned at the beginning of this section, when we increase $q_{s r p}$ the Halo orbits are displaced towards the Sun. It is interesting to see how this displacement is projected in the Floquet reference frame.

We consider a nominal Halo orbit where SRP is neglected $\left(q_{s r p}=0\right)$ and computed different Halo orbits for different $q_{s r p}$, where all the orbits have the same orbital period. We use the Floquet reference system around the first Halo orbit, and for each of the 4 periodic orbits with $q_{s r p} \neq 0$ we compute their position in this reference frame. This results are summarized in Figure 5.
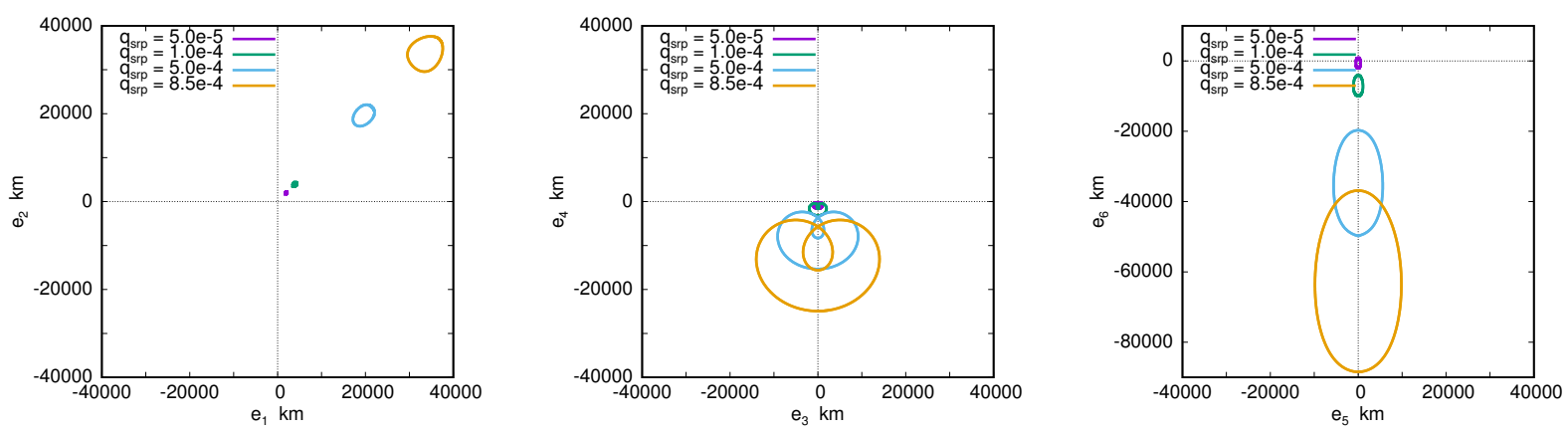

Figure 5. Projection on the saddle, center and neutral Floquet modes of a RTBP Halo orbit (no srp) of the displaced Halo orbit for $q_{s r p}=5 \times 10^{-5}, 10^{-4}, 5 \times 10^{-4}$ and $8.5 \times 10^{-4}$

If we focus on the saddle projection, we notice that all of the displaced periodic orbits are on the top-right quadrant. Moreover, as $q_{s r p}$ increases so does their distance to the origin (i.e. distance to the no SRP Halo orbit). This implies that if we look at the trajectory of two satellites with different $q_{s r p}$ values in the same reference frame, we will see that their saddle behaviors will slightly displaced. 
When we look at the projection of the orbits in the central and neutral planes, in both cases the orbits are displaced along the $\bar{e}_{4}$ and $\bar{e}_{6}$ axis respectively. In the case of the center projection, this means that for two Halo orbits with different $q_{s r p}$ values, the center of rotation for each orbit is slightly displaced. In the case of the neutral direction, variations in $q_{s r p}$ will not have a very drastic effect apart from small changes on their orbital period.

\section{WFIRST trajectory and Station Keeping maneuvers}

The WFIRST mission orbit has been designed to meet requirements specific to its own mission and to remain within the SEL2 environment. With routine MUs and Station keeping maneuvers, WFIRST will be able to maintain its mission orbit for 5 years. In Figure 6 we have a projection of the trajectory of WFIRST. This design was implemented in a MATLAB Module ${ }^{15}$ that uses the Adaptive Trajectory Design ${ }^{16}$ program which allows to find in a systematic way transfer orbits from LEO to SEL1 and SEL2 Halo orbits.

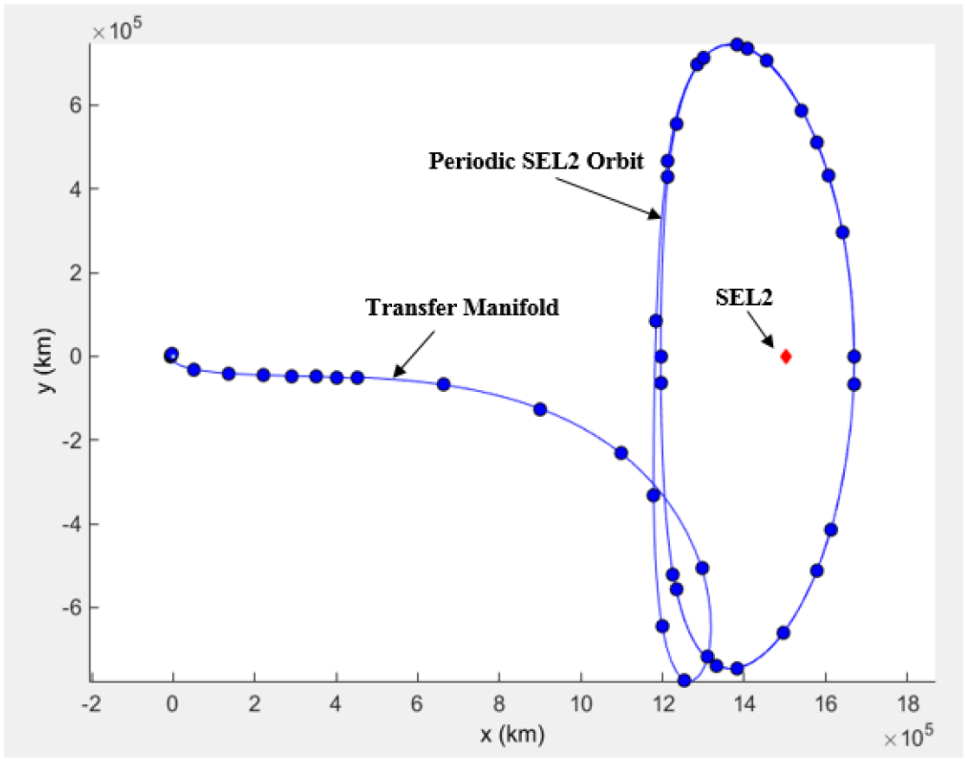

Figure 6. WFIRST trajectory from the Earth to a Halo orbit around SEL2 in the RTBP. ${ }^{15}$

As we have mentioned in the previous section, these Halo orbits are unstable, hence station keeping maneuvers are required to maintain the nominal orbit. These maneuvers are done every 21 days in the direction that cancels out the drift along the unstable manifold. ${ }^{14}$ Additionally, WFIRST will need to perform routine MUs to unload stored momentum in the reaction wheels. The MUs can be modeled as small and random $\Delta v$ 's.

We use the Floquet mode reference frame to compute the required $\Delta v$ for the station keeping maneuvers every 21 days. The idea is to express the trajectory of the satellite in the Floquet mode reference frame $\left\{\bar{e}_{1}(\tau), \ldots, \bar{e}_{6}(\tau)\right\}$ and compute the $\Delta v=\left(0,0,0, d v_{x}, d v_{y}, d v_{z}\right)$ such that:

$$
\phi_{\tau}(x)+\left(0,0,0, d v_{x}, d v_{y}, d v_{z}\right)=c_{2} \bar{e}_{2}(t)+\ldots+c_{6} \bar{e}_{6}(t),
$$

the unstable direction is canceled out. For further details on the implementation see Gomez et al. ${ }^{14}$

We have done a 2 year simulation of the trajectory of a WFIRST around a Halo orbit for $q_{s r p}=10^{-5}$ and a $Z$ amplitude of $120,000 \mathrm{~km}$, performing station keeping maneuvers every 21 days. We have done two simulations, one with MUs every week and another with no MUs. The total $\Delta v$ for these 2 year simulation is of $10.42 \mathrm{~cm} / \mathrm{s}$ when no MUs are considered and of $11.57 \mathrm{~cm} / \mathrm{s}$ when we include the MUs.

Figure 7 shows the projection of the Halo orbit in the Floquet reference frame: saddle projection $\bar{e}_{1}, \bar{e}_{2}$ (left), center projection $\bar{e}_{3}, \bar{e}_{4}$ (middle) and neutral projection $\bar{e}_{5}, \bar{e}_{6}$ (right). The three plots on the top correspond to the simulation with no MUs. We can appreciate how the trajectory rotates around the center projection (middle plot) and on the saddle projection (left plot) how the trajectory escapes along the unstable manifold. Each time there is a $\Delta v$ (i.e. a jump in the phase space) notice that the trajectory comes close 
to the stable direction. The three plots on the bottom correspond to the simulation done with MUs, where we see the general trend of the trajectory is the same as the simulations with no MUs but the trajectory is more disturbed due to the frequent MUs.
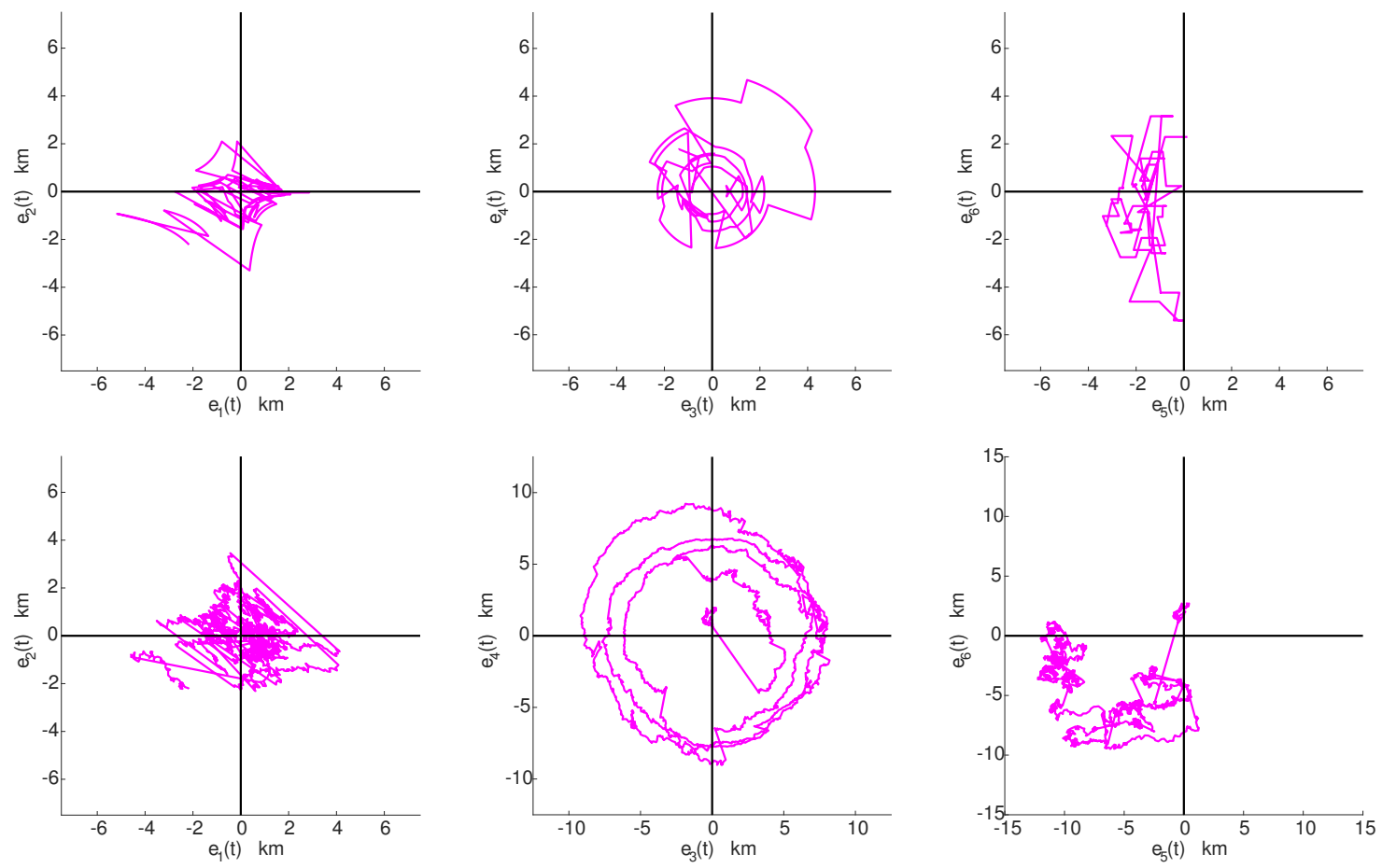

Figure 7. Projection on the saddle, center, neutral Floquet modes of WFIRST trajectory with station keeping maneuvers every 21 days. Top: simulations with no MUs. Bottom: simulation with MUs every week.

\section{WFIRST and Starshade formation flying}

As we have already mentioned, WFIRST's mission orbit is designed to meet its specific mission requirements, and is not to be adjusted to accommodate the Starshade. Starshade will follow a DRM which includes a list of stars and their observation times. During the observations Starshade will remain at a distance of $37,000 \mathrm{~km}$ maintaining a specific geometry so that the Coronagraph can perform its observations. This distance can vary during the transfer of Starshade from one observation to the other, but it must meet the observations geometry at the begin and end of each transfer.

The strategy that we suggest for Starshade in this preliminary study is simple. At the beginning of the simulation Starshade and WFIRST are assumed to be orbiting in the same LPO at SEL2, and at some point Starshade executes a maneuver to separate itself from WFIRST. At that point an initial $\Delta v$ is performed in order to reach at the first observation point $p_{1}$ at time $t_{1}$. Once it reaches the first observation another $\Delta v$ is performed to guarantee that at the end of the observation WFIRST and Starshade meet the required geometry. Once the first observation is finished, another $\Delta v$ is performed to reach the second observation $p_{2}$ at time $t_{2}$. This process is repeated until the end of the DRM. Figure 8 shows a schematic representation of this targeting scheme.

In this paper we will not focus on keeping the relative distance of $37,000 \mathrm{~km}$ and specific geometric configuration during the observations, we just require that this configuration is met at the beginning and at the end of each observations. We will just focus on the transfer trajectories from one observation to another using this Bang-Bang approach. We note that no optimization is made to compute the transfer $\Delta v$. It is the required $\Delta v$ to move Starshade from an initial point $q_{0}$ arrives at $q_{1}$ in time $t_{1}$.

We have considered two different DRMs with 48 observations: the first DRM is very similar to the DRMs that was investigated in a previous study, ${ }^{9}$ where all the observations happened to occur on the Sun-side of the Halo orbit; the second DRM is symmetric to this one where all the simulations are on the anti-Sun-side 


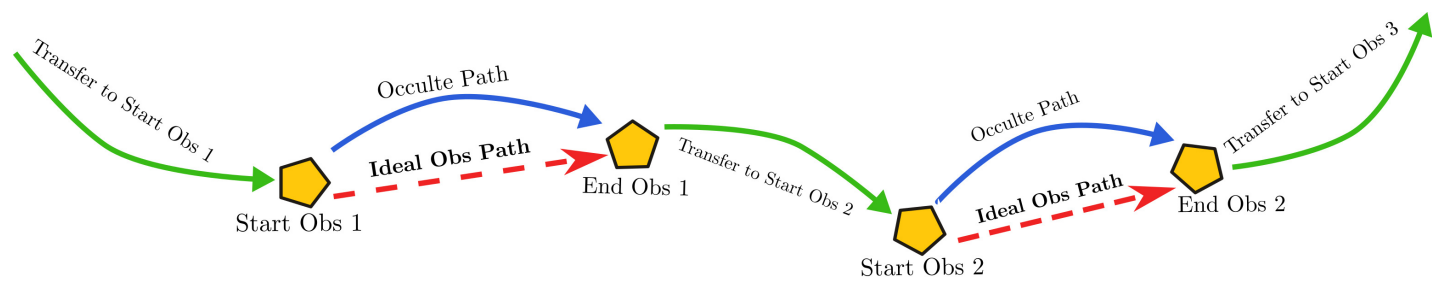

Figure 8. Schematic representation of the Starshade targeting method.

of the Halo orbit. From now on we will refer to these two DRMs as DRM1 and DRM2 respectively. Figure 9 shows the trajectory of WFIRST and Starshade in both DRMs.
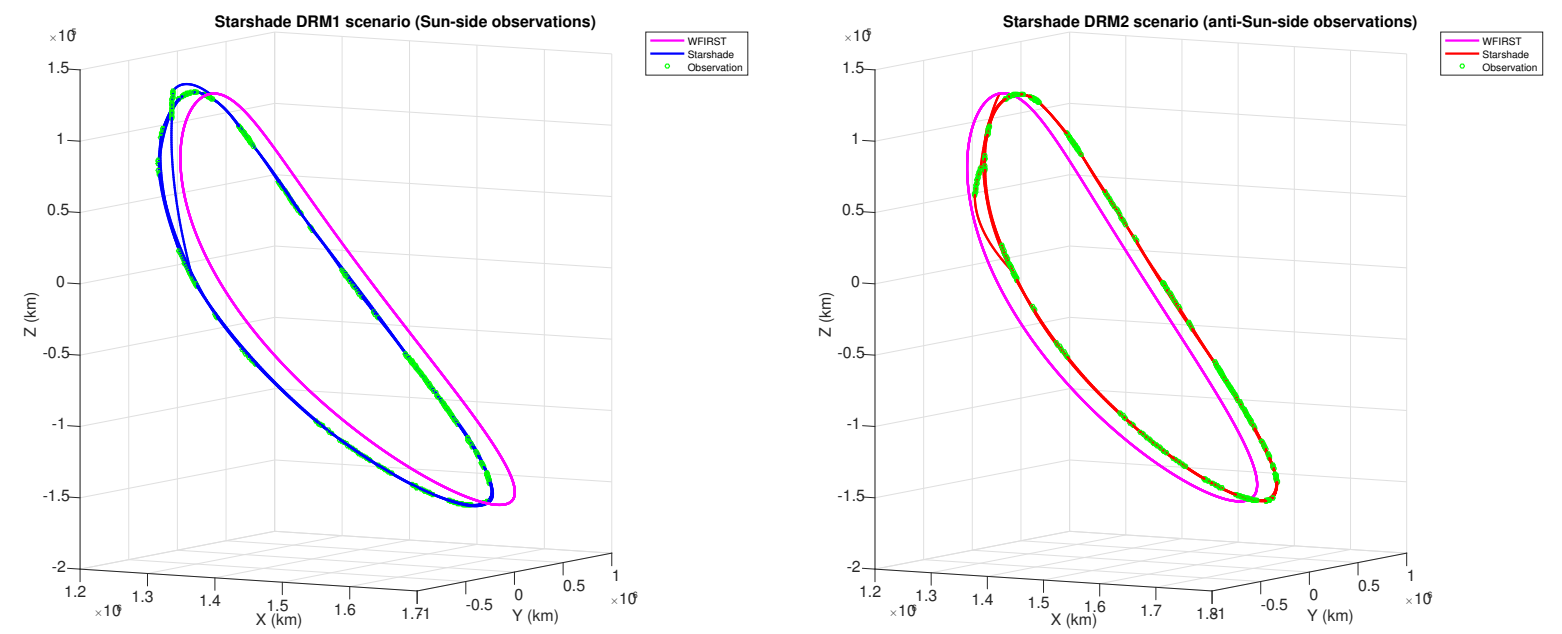

Figure 9. WFIRST and Starshade trajectories for two different DRMs. Left: DRM1 with Sun-side observations (red trajectory). Right: DRM2 with anti-Sun-side observations (blue trajectory). In both plots, the magenta line corresponds to the WFIRST trajectory and the green dots are the different observations in each DRM.

For each DRM we have considered two different SRP models for Starshade: one using a cannonball model, and the other using an N-plane model (with 1 single plate to define the Starshade). In this last case during the transfer between observations, Starshade is always perpendicular to the Sun-Starshade line, and during the observations it is aligned with the WFIRST-Starshade line.

In order to see the relevance of the SRP perturbation we have considered the case when both WFIRST and Starshade have the same SRP coefficient, $q_{s r p}=10^{-5}$ and the case when each probe has a different SRP coefficient, $q_{s r p}=10^{-5}$ for WFIRST and $q_{s r p}=8.5 \times 10^{-4}$ for Starshade. For all the simulations, we have computed the total $\Delta v$ cost to transfer between the 48 observations. We will also look at the projection of the trajectories in the Floquet reference frame to help us understand the results.

\section{A. Same $q_{s r p}$ for WFIRST and Starshade}

In this section, we will discuss the results for the simulations when we consider that both satellites have the same SRP coefficients $\left(q_{s r p}=10^{-5}\right)$. Table 2 summarizes the total $\Delta v$ for the 48 observations in each of the DRMs using both: a cannonball and an N-plate models for SRP effect on the Starshades. As we can see, for both DRMs, there is not much difference between the total $\Delta v$ when we compare the two SRP models. This is probably due to the fact that most of the time Starshade's trajectory is transferring from one observation to another, and during this time Starshade is perpendicular to the Sun-Starshade line, and the SRP approximation in both models is the same. It is possible that if we increase either the number of observations or the observation times, the $\Delta v$ numbers will be affected.

On the other hand, if we compare the total $\Delta v$ for the same SRP model and different DRMs, we can see that in both cases there is a difference of at least $20 \mathrm{~m} / \mathrm{s}$. We recall that in the previous study ${ }^{9}$ a similar 
behavior was observed. In that study, we observed that changing the initial date to start the DRM by a month could result a difference of $40-60 \mathrm{~m} / \mathrm{s}$ in the total $\Delta v$ cost. We can attribute this difference to the same phenomena, essentially that the relative positions of the observation points and WFIRST affects the $\Delta v^{\prime}$ s.

Table 2. Total $\Delta v$ for the 48 observations in DRM1 and DRM2 using a cannonball model and an N-plate model for Starshade.

\begin{tabular}{|l|c|c|}
\hline & Total $\Delta v$ DRM1 & Total $\Delta v$ DRM2 \\
\hline Cannonball Model $\left(q_{s r p}=10^{-5}\right)$ & $772.40 \mathrm{~m} / \mathrm{s}$ & $748.97 \mathrm{~m} / \mathrm{s}$ \\
N-plate Model $\left(q_{s r p}=10^{-5}\right)$ & $771.16 \mathrm{~m} / \mathrm{s}$ & $751.74 \mathrm{~m} / \mathrm{s}$ \\
\hline
\end{tabular}

In Figure 10, we have for each of the simulations, the relation between the observation (x-axis) and the $\Delta v$ required to transfer to that observation. On the left-hand side we have the results for the cannonball SRP model and on the right the N-plane SRP model. The color is used to differentiate between one DRM and the other, where the red lines correspond to DRM1 and the blue lines to DRM2. As we can see for all the cases the $\Delta v$ for each of the transfer orbits is very similar.
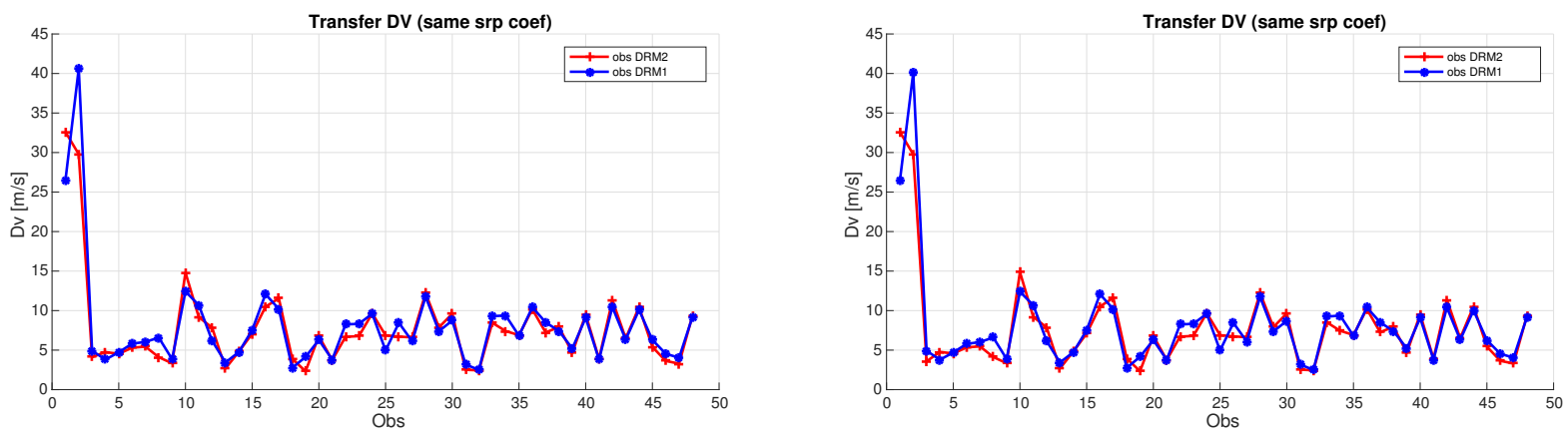

Figure 10. $\Delta v$ to transfer between observations. Red lines DRM1 and blue lines DRM2. Left: cannonball SRP, Right N-plane SRP.

What is interesting is to compare the Starshade trajectory projected on the Floquet mode reference frame. In Figure11 we plot the projection on this reference frame of the trajectories for both DRMs using the Cannonball SRP model (the trajectories in the N-plane model are very similar). We use the same color code as before: the red curve represents DRM1 and blue curve represents DRM2, with the green dots highlighting the parts of the trajectories where the observations take place. As we can see, both projections experience similar behaviors, and are symmetric with respect to the $y$-axis in each reference plane.
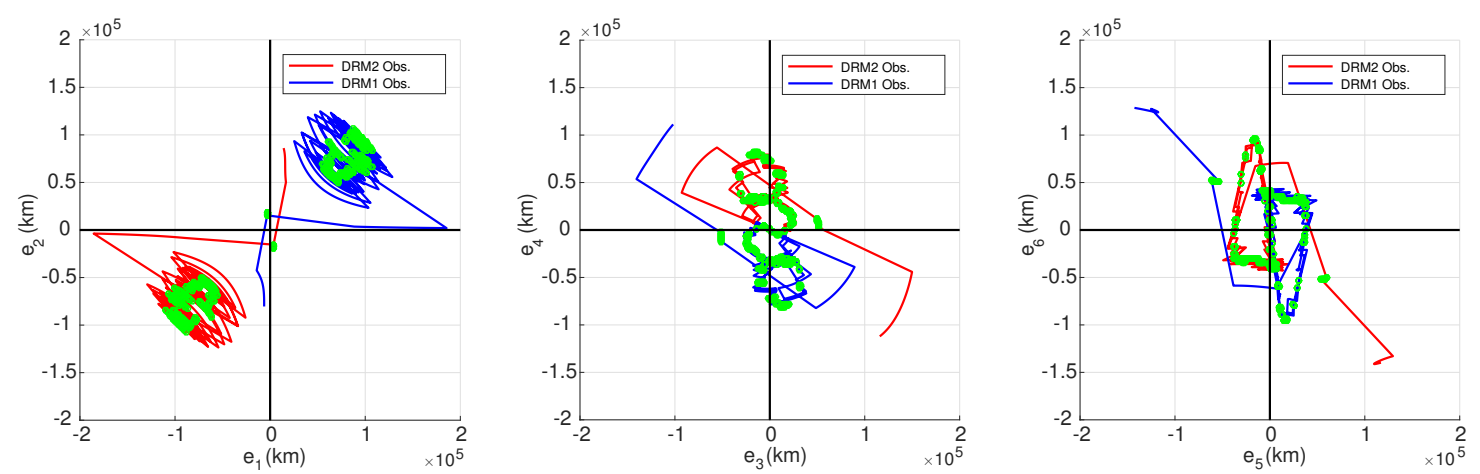

Figure 11. Projection of DRM1 and DRM2 trajectories in the saddle, central and neutral direction. 


\section{B. Different $q_{s r p}$ for WFIRST and Starshade}

Here we summarize the results when we consider different $q_{s r p}$ values for WFIRST and Starshade. We recall that $q_{s r p}=10^{-5}$ for WFIRST and $q_{s r p}=8.5 \times 10^{-4}$ for Starshade. Table 3 shows the total $\Delta v$ for the two different DRMs and the different SRP models for Starshade. Notice that now there is a large difference between the total $\Delta v$ for the two DRMs (Table 3). In both cases, there is a difference of approximately 500 $\mathrm{m} / \mathrm{s}$ between DRM1 (Sun-side observations) and DRM2 (anti-Sun-side observations) where DRM1 is the cheapest option.

Table 3. Total $\Delta v$ for the 48 observations in DRM1 and DRM2 using a cannonball model and an N-plate model for Starshade.

\begin{tabular}{|l|c|c|}
\hline & Total $\Delta v$ DRM1 & Total $\Delta v$ DRM2 \\
\hline Cannonball Model $\left(q_{s r p}=8.5 \times 10^{-4}\right)$ & $541.91 \mathrm{~m} / \mathrm{s}$ & $999.29 \mathrm{~m} / \mathrm{s}$ \\
N-plate Model $\left(q_{s r p}=8.5 \times 10^{-4}\right)$ & $486.71 \mathrm{~m} / \mathrm{s}$ & $1249.38 \mathrm{~m} / \mathrm{s}$ \\
\hline
\end{tabular}

Figure 12 shows for the different simulations the relation between the observation (x-axis) and the required $\Delta v$ to transfer from one observation to the other. These results are consistent with the total cost shown in Table 3 where the transfers between the DRM1 observations are less expensive than the transfers between the DRM2 observations. In order to understand this drastic change between the different observations we must look at the trajectories of both simulations in the Floquet reference frame.
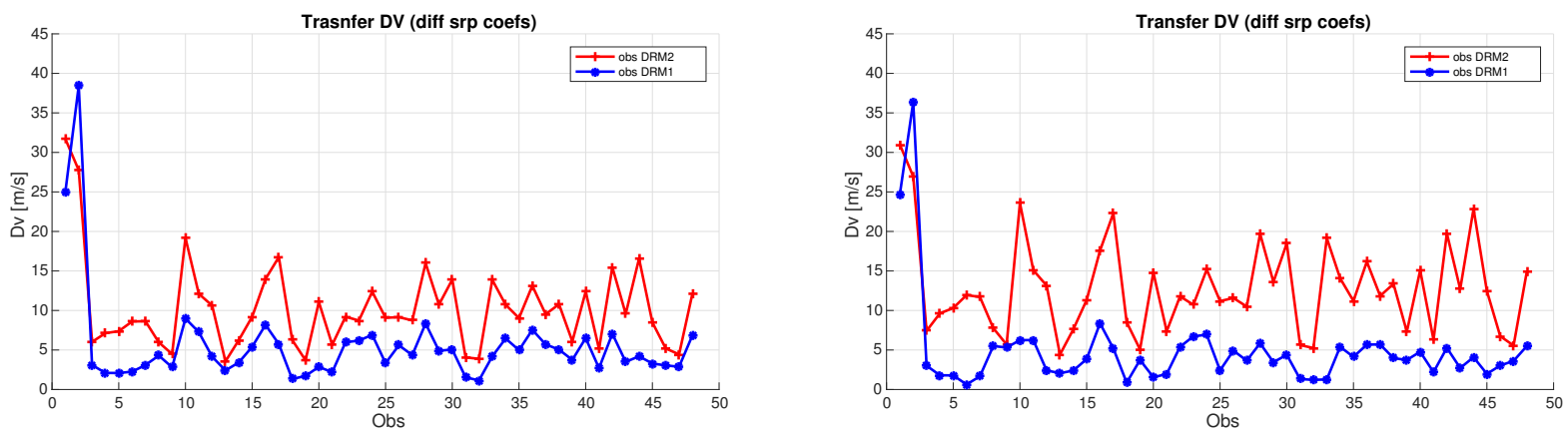

Figure 12. $\Delta v$ to transfer between observations. Red lines DRM1 and blue lines DRM2. Left: cannonball SRP, Right N-plane SRP.

Figure 13 shows the projection of the trajectory for both DRMs on the Floquet mode reference frame. The trajectories shown here correspond to the sumulations where the N-plate model is used to approximate the SRP, but very similar trajectories are observed in for the cannonball approximation. If we compare these trajectories with the ones in Figure 11, we see that the previous symmetry between the two DRM trajectories is broken.
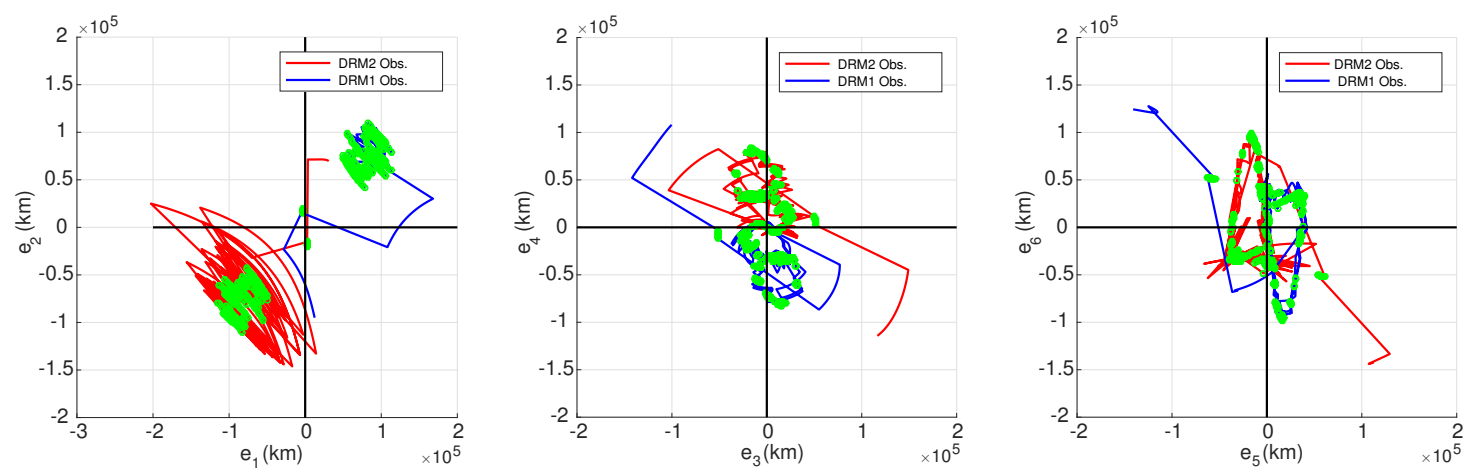

Figure 13. Projection of DRM1 and DRM2 trajectories in the saddle, central and neutral direction.

We recall that in the previous case, both WFIRST and Starshade had the same $q_{s r p}$. So the stable and 
unstable manifolds from WFIRST's reference orbit were governing Starshade's dynamics. Instead, when we consider a different $q_{s r p}$ for Starshade, the periodic orbits have shifted towards the Sun, and as we saw in Figure 5, the projection of these periodic orbits on the saddle plane is shifted approximately $45^{\circ}$. This means that the observations for DRM1 are close to a Halo orbit for Starshade, and the $\Delta v$ to remain there is smaller than for DRM2, where the observation locations are far from the Halo orbit and the rate of expansion due to the saddle makes the total $\Delta v$ increase. This tells us that if we increase $q_{s r p}$ the $\Delta v$ cost for DRM2 can drastically increase.

\section{Transfer time Vs $\Delta v$}

As we have seen, the relative position between Starshade's trajectory and the observations positions in the saddle governing its dynamics is important when determining the total $\Delta v$ budget. This fact was also observed in the previous study, ${ }^{9}$ where we noticed that there where 4 transfer trajectories to certain observations, all with the same transfer time (18 days), and with completely different transfer $\Delta v$ 's. This results showed that there was no relationship between the transfer time and the $\Delta v$, and that under the underlying dynamics must be governing the problem.

In Figure 14 we have a graph that relates each observation with the transfer time (y-axis) and the $\Delta v$ represented by the color of the point. There we can see for all cases that there is no apparent relation between the transfer time and $\Delta v$. A black line has been drawn in order to compare all the observations with 18 days of transfer time. We can also see that there is a difference between the different DRMs and SRP values.
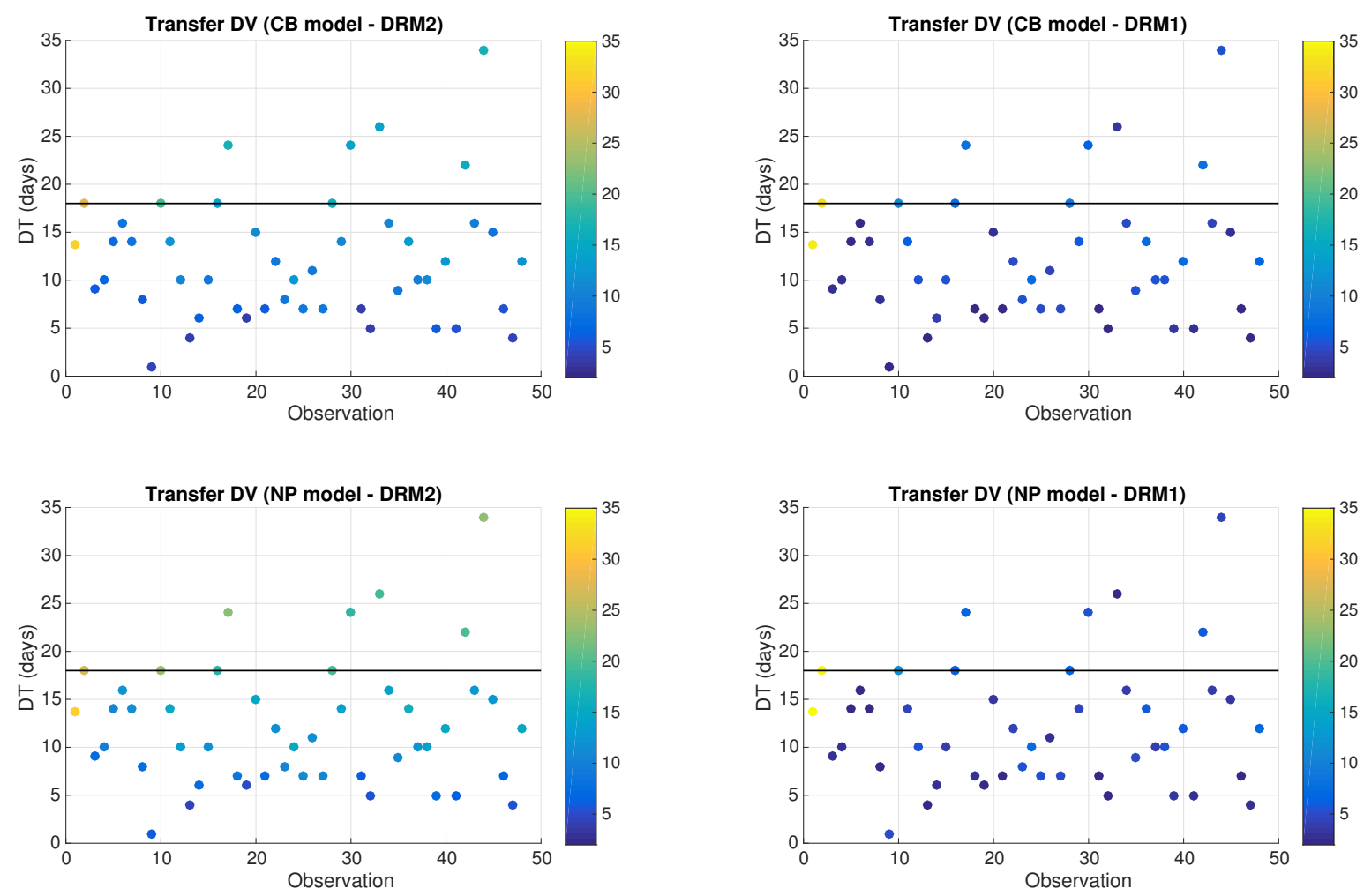

Figure 14. Graph of transfer times. Each dot is one Observations as a function of the of the transfer time from the end of an observation to the beginning of a new one. The $\Delta v$ magnitude in $\mathrm{m} / \mathrm{s}$ is given by the color bar.

It is clear that what really matters when looking at the total $\Delta v$ is the relative position between Starshade and the saddle of the displaced Halo orbit. 


\section{Conclusion}

This paper focuses on understanding the coupled dynamics of WFIRST and Starshade which is designed to follow a prescribed DRM so that WFIRST's Coronagraph can perform high-precision observations. We are interested in understanding the role that SRP plays in the total cost of the mission. As a gravitational model we have considered the RTBP and the SRP effect has been included using either a cannonball model or an N-plate model.

We have seen that the total $\Delta v$ varies drastically depending on the difference between the SRP coefficients $q_{s r p}$ between Starshade and WFIRST. The larger the difference between the area-to-mass ratio between the two probes the more complicated the problem becomes. We have also seen that the relative position of Starshade with respect to to its nominal Halo orbit, the one whose invariant manifolds are leading its trajectory, has an important role on the $\Delta v$ budget.

We have used the Floquet modes as a reference frame around the Halo orbits to describe the dynamics of Starshade during the DRM. This reference frame helps us understand the relation between the $\Delta v$ cost and the relative positions between WFIRST, Starshade and the observation points.

\section{Future Work}

There are still many things that need to be addressed to have a good understanding of the Starshade WFIRST formation. Here are some of the things that we think should be addressed in the near future. As we have seen in both DRM sequences, considering different $q_{\text {srp }}$ coefficients for WFIRST and Starshade (which is a more realistic approach) drastically changed the $\Delta v$ budget. Notice that as the Starshade observations go on, the large $\Delta v$ 's required to go from one formation to another (in most cases these maneuvers are larger that $5 \mathrm{~m} / \mathrm{s}$ ) will make the total mass decrease, affecting the area-to-mass ration and the $q_{s r p}$ coefficient. We propose to look at this into more detail, by performing an analysis where the variation of Starshade's mass is taken into account.

In this paper we have not studied the necessary maneuvers that Starshade should perform to keep the Starshade - WFIRST geometric formation during the whole observation. We have only forced Starshade to match these geometric configurations at the beginning and at the end of each observation having an estimated value of the possible cost. An analysis on the deviation from this configuration during the observations and possible strategies to keep the configuration within a certain regime must be done.

Finally, we are working on including higher-fidelity models for the SRP effects on WFIRST which take into account the shape and reflectivity properties of the satellite. ${ }^{11}$ Such an analysis will affect mainly WFIRST's orbit and possible extensions of its lifetime, but will not have a direct impact of the coupled dynamics. Higher fidelity SRP models can also be used to model the SRP effects on Starshade, but given its shape, a flat plate is a good first approximation and one should focus on having a good estimation of the reflectivity properties of this plate.

\section{Acknowledgments}

The work has been funded under the Goddard Planetary Heliophysics Institute Task 595.001 in collaboration with the University of Maryland Baltimore County (UMBC) under the NNG11PL02A. The attendance to the conference has been supported by the Spanish grant MTM2015-67724-P (MINECO/FEDER).

\section{References}

${ }^{1}$ NASA WFIRST Mission Website, https://wfirst.gsfc.nasa.gov/about.html.

${ }^{2}$ S. Seager, K. Warfield, W. Cash, S. Domagal-Goldman, N. J. Kasdin, M. Kuchner, A. Roberge, S. Shaklam, W. Sparks, M. Thomson, M. Turnbull, D. Lisman, R. Baran, R. Bauman, E. Cady, C. Henegham, S. Martin, D. Scharf, R. Trabert, D. Webb, P. Zarifian, "Exo-S: Starshade Probe-Class Exoplanet Direct Imagining Mission Concept Final Report". https://exoplanets.nasa.gov/stdt/Exo-S_Starshade_Probe_Class_Final_Report_150312_URS250118.pdf, March 2015.

${ }^{3}$ D. Lisman, "WFIRST-Starshade Overview and Introduction to Accommodations Issues". DRM Internal Memo. August 09. 2016 .

${ }^{4}$ L. Millard, K. Howell, "Optimal reconfiguration maneuvers for spacecraft imaging arrays in multi-body regimes." Acta Astronautica, Volume 63, Issues 11-12, pp 1283-1298, December 2008.

${ }^{5} \mathrm{M}$. Ziebart, "High precision analytical solar radiation pressure modelling for GNSS spacecraft", PhD Dissertation, University of East London, 2001. 
${ }^{6}$ B. Rievers, "High precision modelling of thermal perturbations with application to Pioneer 10 and Rosetta", PhD Dissertation, University of Bremen, 2012.

${ }^{7}$ M. Ziebart, P. Dare, "Analytical Solar Radiation Pressure Modelling for GLONASS using a pixel array", Journal of Geodesy, Vol. 75, Issue 11, pp. 587-599, 2001.

${ }^{8}$ M. Ziebart, "Generalized Analytical Solar Radiation Pressure Modeling Algorithm for Spacecraft of Complex Shape", Journal of Spacecraft and Rockets, Volume 41, No. 5, 2004.

${ }^{9}$ C. Webster, D. Folta, "IWSCFF 17-74: Understanding the Sun-Earth Libration Point Orbit Formation Flying Challenges for WFIRST and Starshade", 9th International Workshop on Satellite Constellations and Formation Flying, June 2017, Boulder, CO. 1987.

${ }^{10}$ Milani A., Nobill A.M., Farinella P., "Non-gravitational perturbations and satellite geodesy", Adam Hilger Ltd., Bristol,

${ }^{11}$ A. Farres; D. Folta D.; Webster C.; "Using Spherical Harmonics to model Solar Radiation Pressure Accelerations", AAS/AIAA Astrodynamics Specialist Conference, Columbia River Gorge, Stevenson, WA, August 20-24, 2017.

${ }^{12}$ A. Farres, A.; Jorba, À.; "Periodic and Quasi-Periodic Motion of a Solar Sail close to SL1 in the Earth-Sun System", Celestial Mechanics and Dynamical Astronomy, Volume 107, num. 1-2, pp. 233-253, June 2010.

${ }^{13}$ A., Farres; "Catalogue on the Dynamics of a Solar Sail around L1 and L2", Proceedings of the 4th International Symposium on Solar Sailing, Kyoto, Japan, 17-20 January 2017.

14, G. Gòmez, J. Llibre, R. Martinez, C. Simó, "Dynamics and mission design near libration points - Volume I: fundamentals: the case of collinear libration points", World Scientific Monograph Series in Mathematics, Vol. 2, World Scientific, 2001.

${ }^{15}$ N. Bosanac, "Sun-Earth Libration Point Trajectory Design Module Tutorial, Version 1". December 2016.

${ }^{16}$ D. Folta, A. Haapala, T. Pavlak, K. Howell, "Adaptive Trajectory Design (ATD)", GSFC-IRAD https://engineering.purdue.edu/people/kathleen.howell.1/Gallery/Posters/these_images/IRAD_2013.pdf, 2013. 